\title{
Arbitrarily Reconfigurable and Thermadapt
}

\author{
Reversible Two-Way Shape Memory
}

\section{Polythiourethane Accomplished by Multiple}

\section{Dynamic Covalent Bonds}

Junjie Zhou, ${ }^{1}$ Huimin Yue, ${ }^{1}$ Miaoming Huang, ${ }^{1 *}$ Chaobo Hao, ${ }^{1}$ Suqin He, ${ }^{1}$ Hao Liu, ${ }^{1}$ Wentao Liu, ${ }^{1}$ Chengshen Zhu, ${ }^{1}$ Xia Dong, ${ }^{2,3 *}$ and Dujin Wang ${ }^{2,3}$

${ }^{1}$ School of Materials Science and Engineering, Zhengzhou University, Zhengzhou 450001, P. R. China

${ }^{2}$ CAS Key Laboratory of Engineering Plastics, Beijing National Laboratory for Molecular

Sciences, Institute of Chemistry, Chinese Academy of Sciences, Beijing 100190, P. R. China

${ }^{3}$ University of Chinese Academy of Sciences, Beijing 100049, P.R. China

Corresponding Authors

*E-mail: mmhuang@zzu.edu.cn (Miaoming Huang).

*E-mail: xiadong@iccas.ac.cn (Xia Dong). 
Table S1. The Composition of PTU Networks.

\begin{tabular}{ccccc}
\hline Samples & $\begin{array}{c}\text { Hard segment } \\
\text { content } / \mathrm{wt} \%\end{array}$ & PCL /mol & HDI /mol & PTME /mol \\
\hline PTU-20\% & 20 & 0.005 & 0.016 & 0.0049 \\
PTU-30\% & 30 & 0.005 & 0.024 & 0.0091 \\
PTU-40\% & 40 & 0.005 & 0.036 & 0.015 \\
PTU-50\% & 50 & 0.005 & 0.053 & 0.023 \\
\hline
\end{tabular}

The PTU contains two segments, including soft segment (PCL) and hard segment (HDI and PTME). By fixing the dosage of PCL diol (20g) and changing the dosage of HDI and PTME, PTU with different hard segment content can be obtained. The Equations S1 and S2 were used to determine the composition of the PTU.

$$
\begin{gathered}
R=\frac{n[\mathrm{NCO}]}{n[\mathrm{OH}]+n[\mathrm{SH}]}=\frac{\frac{m_{1}}{168.19} \times 2}{\frac{20}{4000} \times 2+\frac{m_{2}}{488.66} \times 4}=1.05 \\
H_{\mathrm{S}}=\frac{m_{1}+m_{2}}{20+m_{1}+m_{2}}
\end{gathered}
$$

where $R$ represents the group ratio. The isocyanate group is a slight excess, as it is prone to side reactions (reaction with water in the air). $m_{1}$ and $m_{2}$ represents the mass of HDI and PTME, respectively. $H_{\mathrm{s}}$ stands for weight percentage of hard segment. 


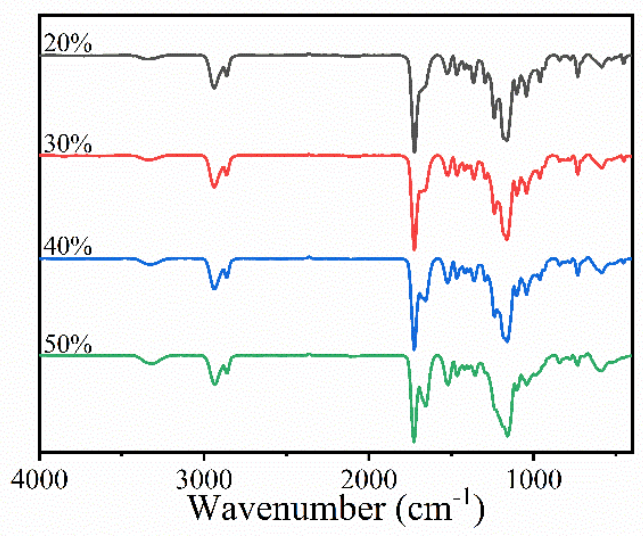

Figure S1. FTIR curves of the PTU samples.

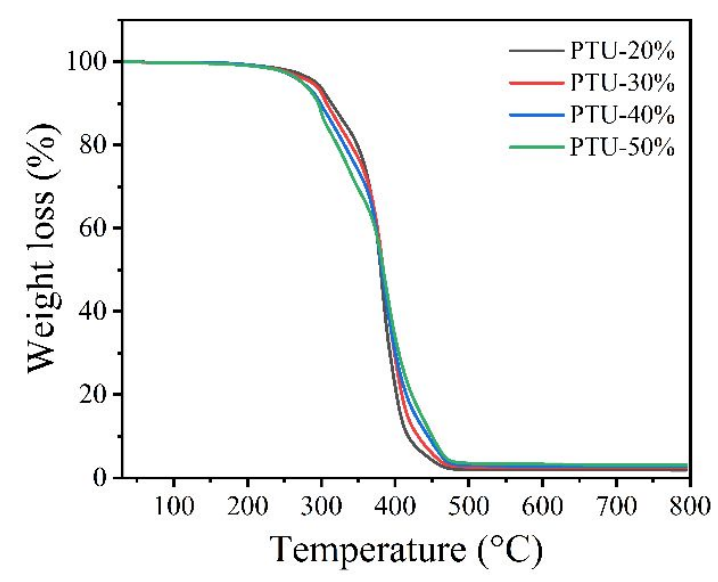

Figure S2. TGA curves of the PTU samples.

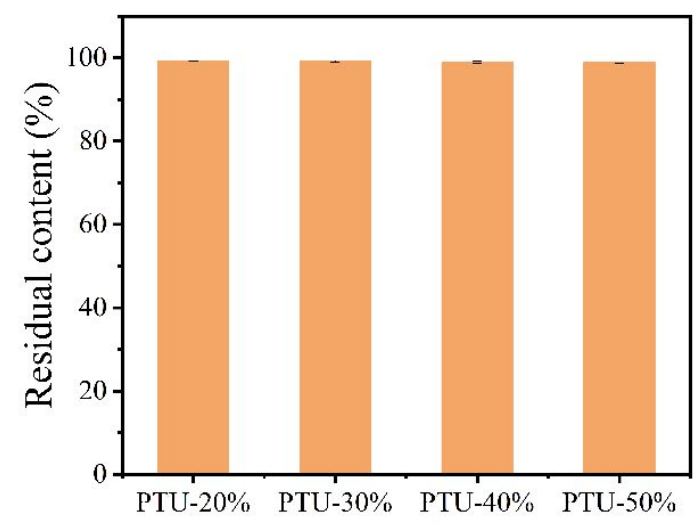

Figure S3. Residual content of PTU samples after immersed in water for $300 \mathrm{~h}$ at room temperature. 


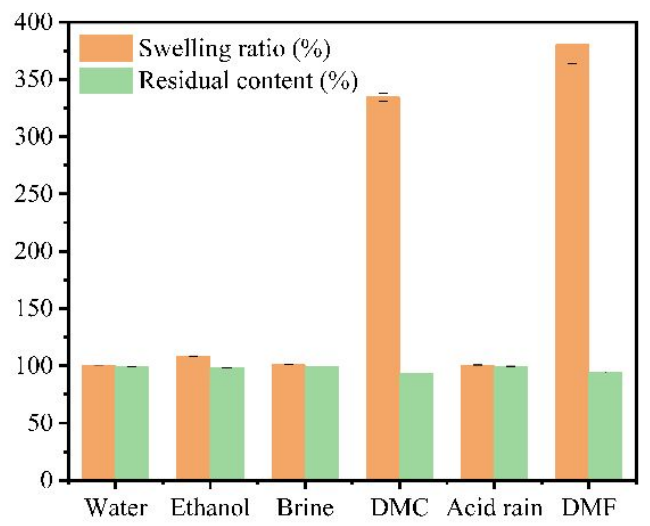

Figure S4. Swelling ratios and residual content of PTU samples in different solvents for $72 \mathrm{~h}$ at room temperature.

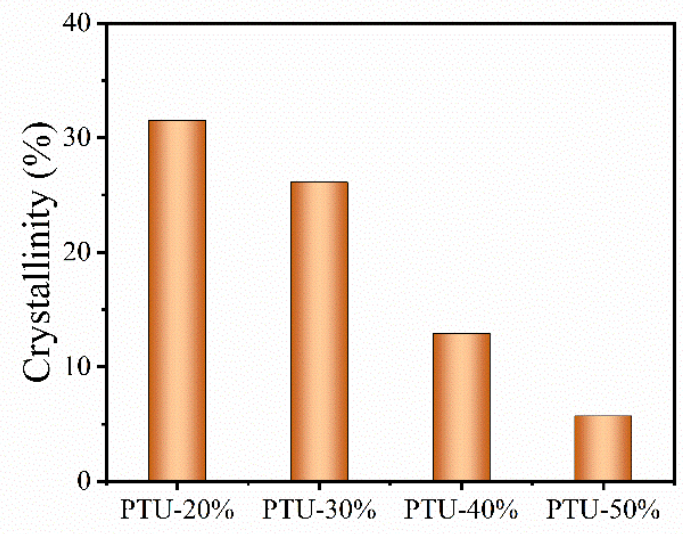

Figure S5. Crystallinity of the PTU samples obtained from XRD data.

The calculation equation of cross-linking density $(v)$ is shown as follows:

$$
v=E / 3 R T
$$

where $E$ refers to the tensile storage modulus of the sample characterized by DMA, $R$ refers to the gas constant, and $T$ is $393.2 \mathrm{~K}$. 
(a)

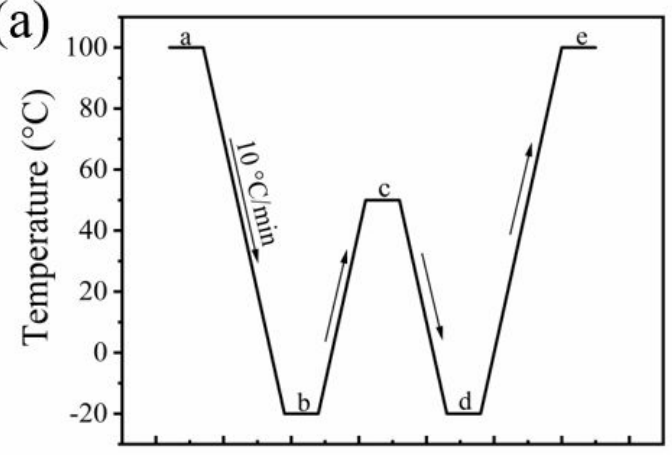

Time (min)

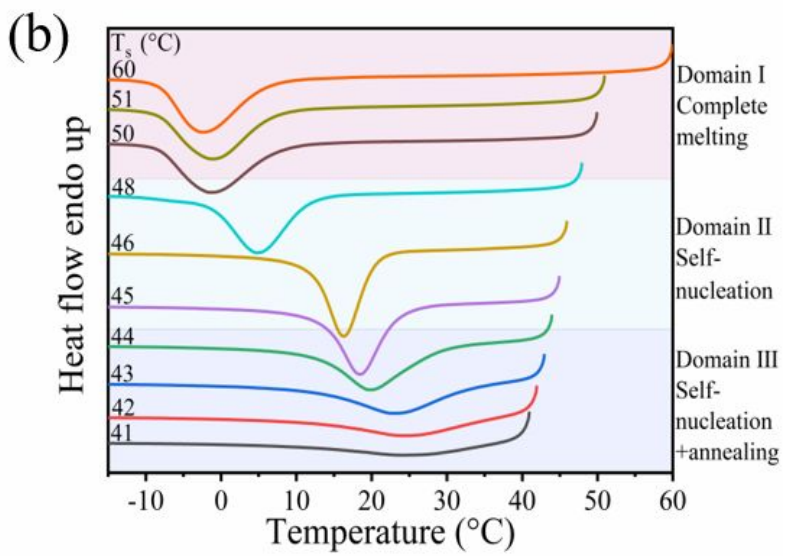

Figure S6. (a) Schematic diagram of temperature control of self-nucleated crystallization (SNC).

a. Erasure of thermal history. b. "Standard" semi-crystalline state. c. Partial melting and annealing. d. SNC-treated sample. e. Complete melting. (b) DSC cooling curves (the process from $\mathrm{c}$ to d) of PTU-20\% after treating at different self-nucleation temperature $\left(\mathrm{T}_{\mathrm{s}}\right)$ for $5 \mathrm{~min}$.

Table S2. Reversible Two-Way Shape Memory Parameters of PTU-20\% Sample.

\begin{tabular}{cccccc}
\hline & $1^{\text {st }}$ cycle & $2^{\text {nd }}$ cycle & $3^{\text {rd }}$ cycle & $4^{\text {th }}$ cycle & Average $/ \%$ \\
\hline $\begin{array}{c}\text { Shape recovery } \\
\text { ratio } / \%\end{array}$ & 85.6 & 88.3 & 90.4 & 92.0 & 89.1 \\
$\begin{array}{c}\text { reversible strain } \\
\text { fraction } / \%\end{array}$ & 25.3 & 22.8 & 21.3 & 20.0 & 22.4 \\
\hline
\end{tabular}




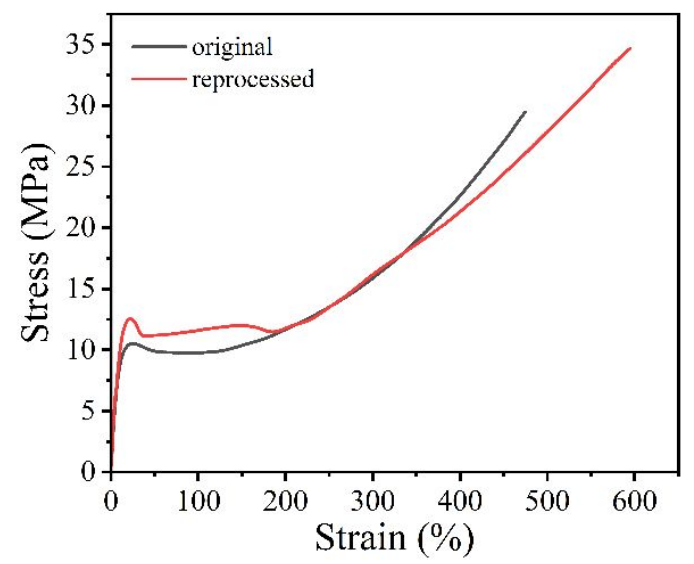

Figure S7. The tress-strain curves of PTU-20\% film before and after reprocessing.

Table S3. The Performance of PTU Samples before and after Reprocessing.

\begin{tabular}{cccccc}
\hline \multicolumn{2}{c}{ Samples } & $\begin{array}{c}\text { Tensile strength } \\
\text { /MPa }\end{array}$ & $\begin{array}{c}\text { Elongation at } \\
\text { break } / \%\end{array}$ & $v / \mathrm{mol}^{2} / \mathrm{m}^{3} \mathrm{a}$ & $X_{\mathrm{c}} / \% \mathrm{~b}$ \\
\hline \multirow{2}{*}{ PTU-20\% } & original & $29.7 \pm 1.1$ & $474.8 \pm 7.5$ & 356.1 & 31.5 \\
& reprocessed & $34.5 \pm 1.3$ & $571.7 \pm 44.0$ & 354.7 & 34.7 \\
\multirow{2}{*}{ PTU-30\% } & original & $21.2 \pm 0.8$ & $251.8 \pm 10.1$ & 579.9 & 26.1 \\
& reprocessed & $26.5 \pm 2.1$ & $341.2 \pm 26.4$ & 438.8 & 26.8 \\
PTU-40\% & original & $18.9 \pm 2.7$ & $198.4 \pm 20.8$ & 778.1 & 12.9 \\
& reprocessed & $25.2 \pm 1.6$ & $229.4 \pm 14.7$ & 619.1 & 16.5 \\
\hline
\end{tabular}

${ }^{\mathrm{a}}$ Cross-linking density calculated from DMA; ${ }^{\mathrm{b}}$ Crystallinity calculated from XRD.

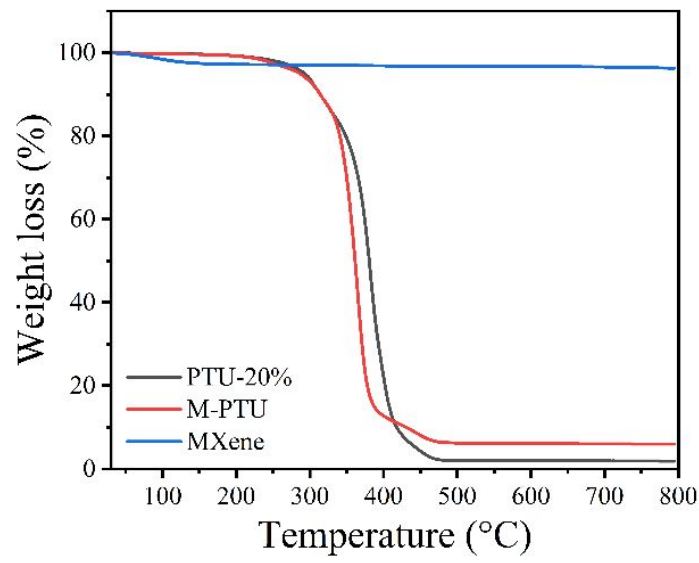

Figure S8. TGA curves of the samples. 

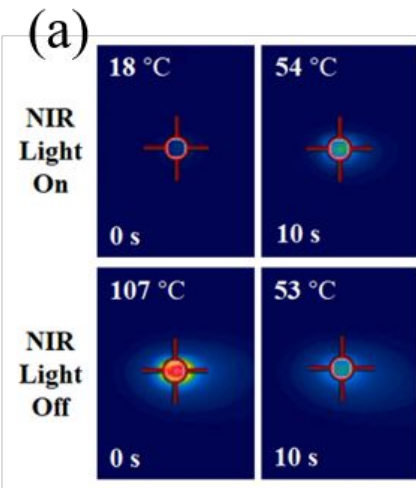

(c)

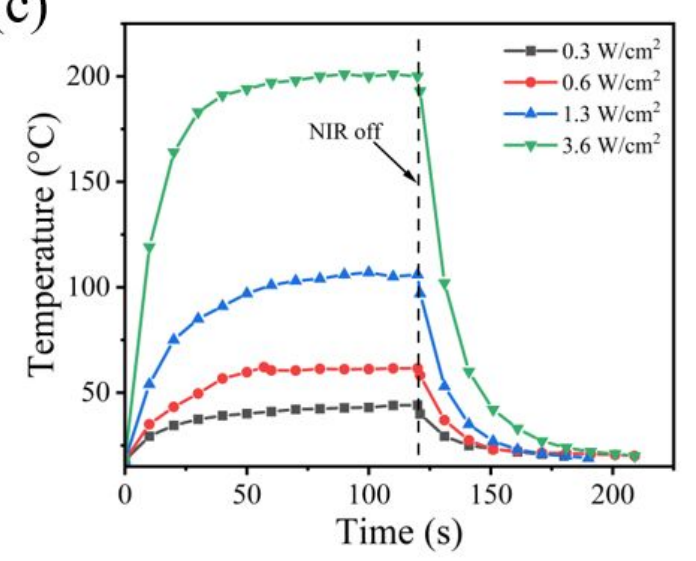

(b)
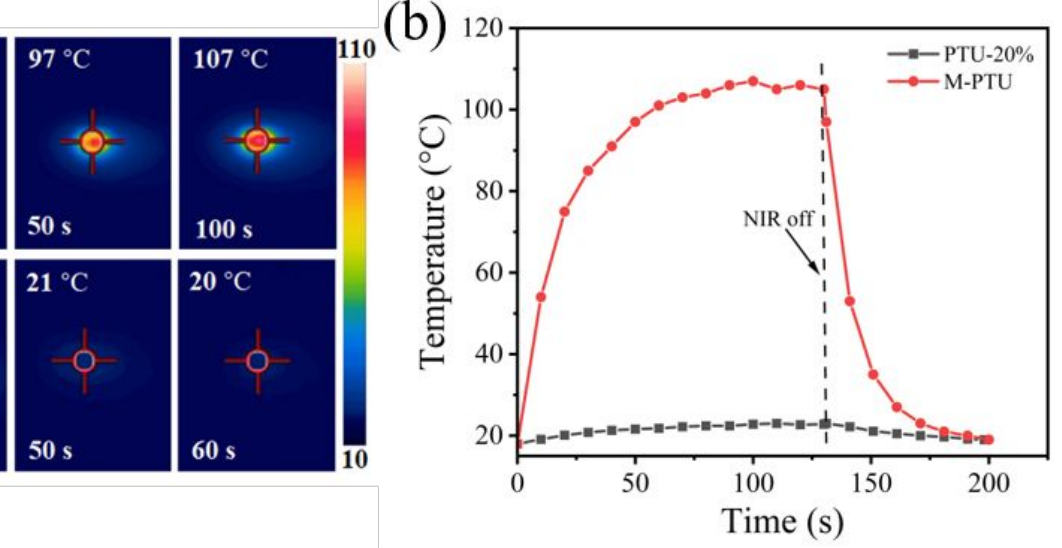

(d)

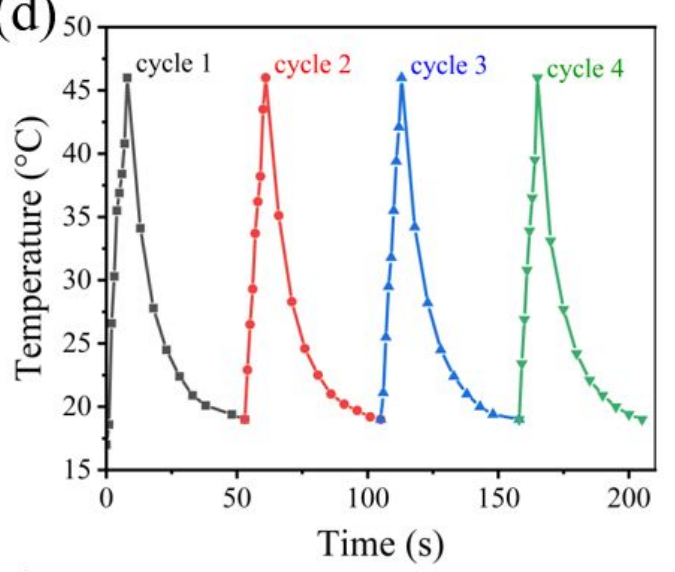

Figure S9. Temperature changes as a function of time while the NIR light is turned on and off.

(a) Thermal-infrared images of M-PTU composite under NIR irradiation $\left(808 \mathrm{~nm}, 1.3 \mathrm{~W} / \mathrm{cm}^{2}\right)$.

(b) Temperature changes of M-PTU composite and PTU at the same light source (808 nm, 1.3

$\mathrm{W} / \mathrm{cm}^{2}$ ). (c) Temperature changes of M-PTU composite irradiated under different power densities. (d) Four cycles of temperature change of M-PTU composite at a constant light intensity $\left(808 \mathrm{~nm}, 1.3 \mathrm{~W} / \mathrm{cm}^{2}\right)$. 